\title{
Noninvasive imaging of prefrontal activation during attention-demanding tasks performed while walking using a wearable optical topography system
}

\author{
Hirokazu Atsumori \\ Masashi Kiguchi \\ Takusige Katura \\ Tsukasa Funane \\ Akiko Obata \\ Hiroki Sato \\ Hitachi, Ltd. \\ Advanced Research Laboratory \\ 2520 Akanuma, Hatoyama \\ Saitama 350-0395, Japan
}

\section{Takaaki Manaka}

Mitsumasa Iwamoto

Tokyo Institute of Technology

Department of Physical Electronics

2-12-1 O-okayama, Meguro-ku

Tokyo 152-8552, Japan

\author{
Atsushi Maki \\ Hideaki Koizumi \\ Kisou Kubota \\ Hitachi, Ltd. \\ Advanced Research Laboratory \\ 2520 Akanuma, Hatoyama \\ Saitama 350-0395, Japan
}

\begin{abstract}
Optical topography (OT) based on near-infrared spectroscopy is a noninvasive technique for mapping the relative concentration changes in oxygenated and deoxygenated hemoglobin (oxy- and deoxy- $\mathrm{Hb}$, respectively) in the human cerebral cortex. In our previous study, we developed a small and light wearable optical topography (WOT) system that covers the entire forehead for monitoring prefrontal activation. In the present study, we examine whether the WOT system is applicable to OT measurement while walking, which has been difficult with conventional OT systems. We conduct OT measurements while subjects perform an attention-demanding (AD) task of balancing a ping-pong ball on a small card while walking. The measured time course and power spectra of the relative concentration changes in oxy- and deoxy-Hb show that the step-related changes in the oxy- and deoxy- $\mathrm{Hb}$ signals are negligible compared to the taskrelated changes. Statistical assessment of the task-related changes in the oxy-Hb signals show that the dorsolateral prefrontal cortex and rostral prefrontal area are significantly activated during the AD task. These results suggest that our functional imaging technique with the WOT system is applicable to OT measurement while walking, and will be a powerful tool for evaluating brain activation in a natural environment. (๑) 2010 Society of Photo-Optical Instrumentation Engineers. [DOI: 10.1117/1.3462996]
\end{abstract}

Keywords: wearable optical topography; walking; attention.

Paper 09373RR received Aug. 25, 2009; revised manuscript received Apr. 30, 2010 accepted for publication May 12, 2010; published online Jul. 20, 2010

\section{Introduction}

Around the turn of the century, near-infrared spectroscopy (NIRS) was used for monitoring cortical activation noninvasively to measure changes in hemoglobin concentration. ${ }^{1-4}$ This technique may provide change in the product of the concentration $(C)$ and effective optical path length $(L)$ for oxygenated hemoglobin (oxy-Hb) and deoxygenated hemoglobin $\left(\right.$ deoxy-Hb) in the cerebral cortex: $\Delta\left(C_{\text {oxy }} \cdot L\right)$ and $\Delta\left(C_{\text {deoxy }} \cdot L\right)$, respectively. These changes $\left[\Delta\left(C_{\mathrm{oxy}} \cdot L\right)\right.$ and $\left.\Delta\left(C_{\text {deoxy }} \cdot L\right)\right]$ are referred to as oxy-Hb signals $\left(\Delta C_{\text {oxy }}^{\prime}\right)$ and deoxy-Hb signals $\left(\Delta C_{\text {deoxy }}^{\prime}\right)$, respectively. Optical topography (OT) based on an NIRS technique has been developed as a noninvasive technique for mapping $\Delta C_{\mathrm{oxy}}^{\prime}$ and $\Delta C_{\text {deoxy }}^{\prime}$ with multiple measurement points. ${ }^{5-8}$ In this technique, $\Delta C_{\text {oxy }}^{\prime}$ and $\Delta C_{\text {deoxy }}^{\prime}$ in the cerebral cortex are measured at multiple points by using alternating optical fiber bundles for irradiation and detection with light at two different wavelengths. OT systems have been used for assessing the cognitive functions of infants $^{9-12}$ and for clinical applications ${ }^{13,14}$ because they are

Address all correspondence to: Hirokazu Atsumori, Advanced Research Laboratory, Hitachi, Ltd., 2520 Akanuma, Hatoyama, Saitama 350-0395, Japan. Tel: 81-49-296-6111; Fax: 81-49-296-5999; E-mail: hirokazu.atsumori.et@ hitachi.com noninvasive and less constraining for subjects than other functional imaging techniques, such as functional magnetic resonance imaging and positron emission tomography. OT systems have been used in studies on brain functions related to human gait ${ }^{15-17}$ and balance control, ${ }^{18}$ as they do not constrain a subject's posture. The studies on human gait have revealed that walking and running on a treadmill are associated with activations in the medial sensorimotor cortices and the supplementary motor area, ${ }^{15}$ the prefrontal cortex, ${ }^{16,17}$ and the premotor cortex, ${ }^{16}$ and a study with an apparatus for perturbing human balance revealed the role of the prefrontal cortex in human balance control. ${ }^{18}$ These studies effectively changed the parameters and conditions in their experiments on human walking and balance control on a treadmill or apparatus. However, conventional OT systems have required that a subject's head position does not move beyond the length of the optical fiber bundles in such OT studies. In recent studies, wearable NIRS or OT systems have been reported and were successful in freeing up the subject's head position. ${ }^{19-21}$ However, they had two to eight measurement points covering a region that was much less than or comparable to half of the forehead.

We developed in our previous study a wearable optical topography (WOT) system that has 22 measurement points

1083-3668/2010/15(4)/046002/7/\$25.00 @ 2010 SPIE 
covering the entire forehead for monitoring activation in the dorsolateral prefrontal cortex (DLPFC) and rostral prefrontal area in humans. ${ }^{22}$ The wearable units of the system are small and light, weighing about $1 \mathrm{~kg}$, so the WOT system enables us to observe activation in a subject's prefrontal area while he/she easily wears the units. Thus, the WOT system has the possibility to be used to study cognitive functions in the large prefrontal area of freely moving subjects, and to assess those of subjects in high risk situations as in airplanes, nuclear areas, or surgery rooms.

To investigate cognitive functions when a subject performs a cognitive task while moving, it is essential to evaluate taskrelated changes in measurement signals without artifacts related to the subject's physical movement. Thus, this study aimed to examine whether the WOT system is applicable to assessment of task-related functions when subjects performed a task while moving. For this examination, the subjects in this study performed an attention-demanding (AD) task that was designed to simplify human behavior in daily life, such as carrying something in a careful way while walking.

\section{Materials and Methods}

\subsection{Wearable Optical Topography System and Optical Topography Measurements}

The WOT system has been developed to be small, light, and wearable. ${ }^{22}$ It consists of a probe unit, a processing unit, and the WOT's computer. The probe unit has a $2 \times 8$ alternating arrangement of irradiation and detection positions covering the entire forehead, with 22 measurement points [Fig. 1(a)]. The separation of each pair of irradiation and detection positions was set to $30 \mathrm{~mm}$, giving the probe unit a coverage area of $30 \times 210 \mathrm{~mm}^{2}$ on the subject's forehead, including the bilateral temples. This arrangement enables us to monitor the cortical activations mainly in the dorsolateral prefrontal cortex (DLPFC) and the rostral prefrontal area. The probe unit has a flexible pad with irradiation and detection positions to fit the subject's head [Fig. 1(b)]. Vertical-cavity surface emitting laser diodes with two wavelengths (790 and $850 \mathrm{~nm})$ were used as light sources to emit light onto the scalp at each irradiation position, and silicon photodiodes were located at each detection position to detect multiply-scattered light in the biological tissue.

The processing unit is connected to the probe unit through a flexible cable bundle for controlling OT measurements and accumulating 5-Hz sampling data into a flash memory inside the processing unit. The processing unit also sends data to the WOT's computer via wireless communication connections, the maximum distance of which is about $100 \mathrm{~m}$. Measurement data are constantly recorded into the flash memory to avoid lack of data by disconnection and are loaded by the WOT's computer. The probe and processing units, which are respectively worn on the subject's head and waist, weigh about $1 \mathrm{~kg}$. Thus, these units are easily carried by healthy adults, which means that the WOT system enables measurement in more natural conditions than possible with conventional systems.

The WOT's computer sends configuration information to the processing unit and receives OT measurement data from the unit, which can then be displayed and analyzed. In this study, we used a modified WOT system that has laser diodes

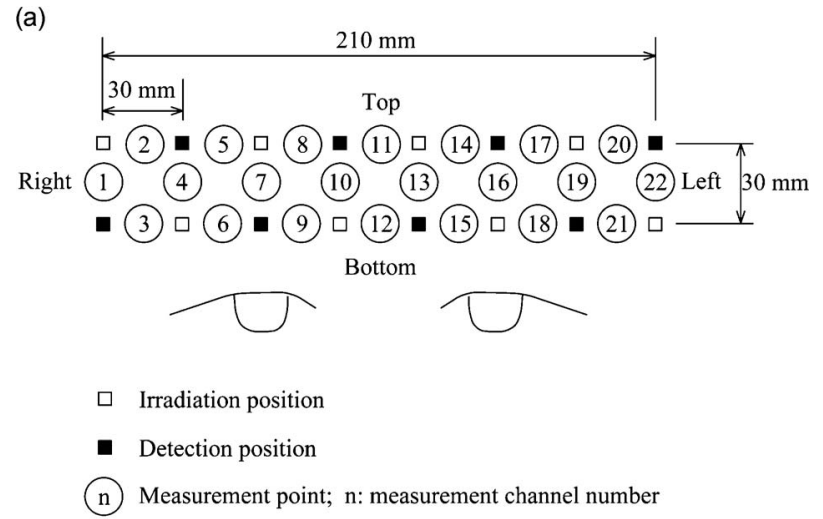

(b)

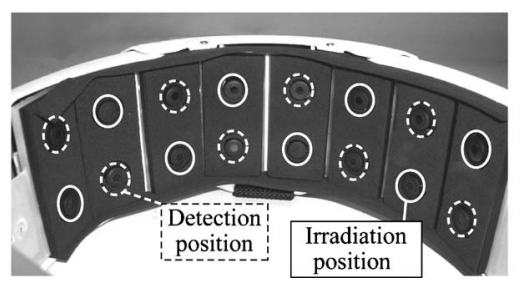

Fig. 1 (a) Configuration of irradiation and detection positions and of measurement points in the probe unit. White and black squares represent irradiation and detection positions, respectively. White circles represent measurement points; numbers are measurement channel numbers. The probe unit has a $2 \times 8$ alternating arrangement of irradiation and detection positions with 22 measurement points. The separation of each pair of irradiation and detection positions was set to $30 \mathrm{~mm}$, giving the probe unit a coverage area of $30 \times 210 \mathrm{~mm}^{2}$ on the subject's forehead. (b) Photograph of the probe unit. The probe unit has a flexible pad with eight irradiation positions and eight detection positions. The probe unit can be worn on the subject's head.

with two wavelengths (754 and $830 \mathrm{~nm}$ ) as light sources in the probe unit, because Sato et al. suggested that noise levels in $\mathrm{Hb}$ changes decreased when using wavelengths shorter than $782 \mathrm{~nm}$ paired with $830 \mathrm{~nm}$, although the detected power appears to be weaker the shorter the wavelength. ${ }^{23}$ We selected $754 \mathrm{~nm}$ from such a wavelength range for optimizing our system in consideration of signal-to-noise ratio and the level of detected power.

OT measurements for behavioral tasks were executed with the modified WOT system and a personal computer (PC) for presenting sounds (see Fig. 2). The PC memorized a proce-

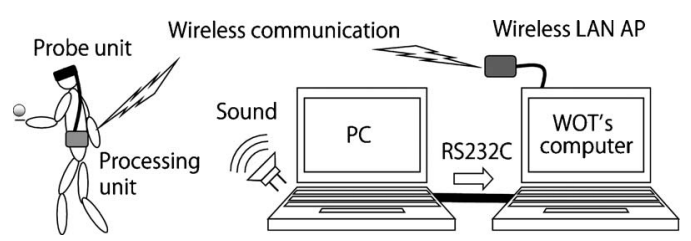

Fig. 2 Experimental setup of OT measurements for behavioral task. The subjects wore the probe unit on their heads and the processing unit on their waists. The PC presented a sound to show the subjects the walking and turning pace. It also sent trigger signals to start measurement, change the condition, and stop the measurement to the WOT's computer. The WOT's computer sent configuration information to the processing unit and received OT measurement data from the unit via wireless communication connections. 
Task

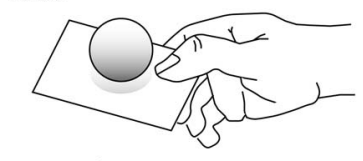

Control

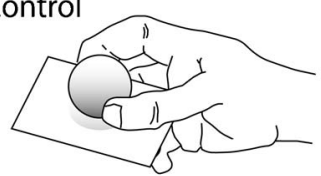

(a)

Walk (10 s)
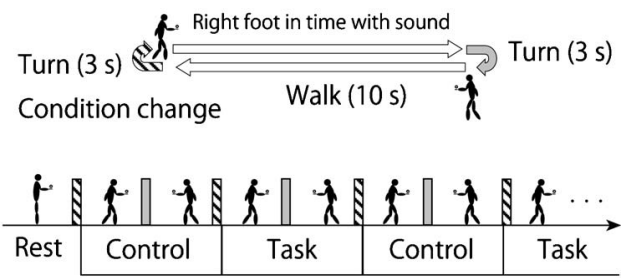

(c)

Fig. 3 Behavioral task and procedure. (a) Ball-carrying tasks while walking with two conditions: task condition, where a subject balances a ball on a card held between his/her right thumb and index finger, and control condition, where the subject holds the ball between his/ her right thumb and index finger as it rests on the card in his/her hand. (b) Subject's eye direction in behavioral task. Subjects were required to gaze at the ball from the start to the end of an experimental session. (c) Task procedure. Subjects stood still in the rest period for about $20 \mathrm{~s}$, then by stepping with the right foot in time to a sound presented every $1 \mathrm{~s}$ by a PC, walked straight for $10 \mathrm{~s}$ and executed a turn over $3 \mathrm{~s}$. They then walked in the return direction for $10 \mathrm{~s}$ at the same pace and changed the way the ball was carried during the next turn executed over $3 \mathrm{~s}$.

dure for presenting sounds from the start to the end of each measurement. The WOT's computer and the PC were connected with an RS232C cable, through which the PC sent trigger signals to the WOT's computer for starting measurement, changing conditions, and stopping measurement.

While the subjects performed the behavioral tasks, we measured the oxy- and deoxy-Hb signals in their prefrontal cortices using the WOT system. The subjects wore the probe unit on their heads and the processing unit on their waists. Gain values for digital potentiometers in the probe unit were optimized depending on the detected light power before the OT measurements.

\subsection{Behavioral Task}

The behavioral task performed by the subjects while walking was an $\mathrm{AD}$ task that requires executive functions in natural walking. This was a ball-carrying task with two conditions (task and control conditions, see Fig. 3), which were controlled by sounds presented by a PC. A session including the two conditions commenced when a warning sound was presented by the PC. For each condition, the subjects performed two sets of alternating walking and turning periods. In the first walking period of $10 \mathrm{~s}$, a low-pitched sound was presented every second by the PC, and the subject stepped with his/her right foot hitting the ground in time with the sound while balancing a ball on a card or holding a ball in the task and control conditions, respectively. The sound presented by the PC produced a 2-Hz step by both feet as the subjects' walking pace. When a high-pitched sound was presented at every second in a turning period of $3 \mathrm{~s}$, the subjects turned around at the same walking pace. After walking in the return direction in the second walking period of $10 \mathrm{~s}$, the subjects changed the way the ball was carried, alternating the condition. Each session included five task conditions and six control conditions, where the subjects were required to gaze at the ball while performing the ball-carrying task. In this task paradigm, subjects consistently stepped with their feet hitting the ground at the same pace from the start to the end of the experiments. Thus, we considered the control condition period as a walking period without the AD task, and the task condition period as that with the AD task.

OT measurements for the behavioral task were conducted in a gymnastic hall that was large enough for subjects to walk in both directions. Before OT measurements, subjects attended a practice session, including two pairs of the task and control conditions, so the subjects could be familiar with the ball-carrying task. In the OT measurement, only one subject dropped the ball during the first task condition period and restarted the experiment.

\subsection{Subjects}

Six healthy adults (4 males and 2 females aged between 25 and 34 years, mean age of $29.7 \pm 3.3 \mathrm{SD}$ ) participated in this study. All were right-handed and had no neurological abnormalities. Informed consent was obtained from each subject.

\subsection{Data Analysis}

To analyze the optical data, changes in the product of the concentration and the effective optical path length for oxyand deoxy-Hb were calculated using the modified BeerLambert law: ${ }^{5}$

$$
\begin{aligned}
\Delta\left(C_{\mathrm{oxy}} \cdot L\right)= & \Delta C_{\mathrm{oxy}(\lambda 1, \lambda 2)}^{\prime}=\left[-\varepsilon_{\operatorname{deoxy}(\lambda 2)} \cdot \Delta A_{(\lambda 1)}\right. \\
& \left.+\varepsilon_{\operatorname{deoxy}(\lambda 1)} \cdot \Delta A_{(\lambda 2)}\right] / E \\
\Delta\left(C_{\mathrm{dexy}} \cdot L\right)= & \Delta C_{\mathrm{deoxy}(\lambda 1, \lambda 2)}^{\prime}=\left[\varepsilon_{\mathrm{oxy}(\lambda 2)} \cdot \Delta A_{(\lambda 1)}\right. \\
& \left.-\varepsilon_{\mathrm{oxy}(\lambda 1)} \cdot \Delta A_{(\lambda 2)}\right] / E,
\end{aligned}
$$

where

$$
E=\varepsilon_{\operatorname{deoxy}(\lambda 1)} \cdot \varepsilon_{\operatorname{oxy}(\lambda 2)}-\varepsilon_{\operatorname{deoxy}(\lambda 2)} \cdot \varepsilon_{\operatorname{oxy}(\lambda 1)} \cdot
$$

The Hb signals $\left(\Delta C_{\text {oxy }}^{\prime}(\lambda 1, \lambda 2)\right.$ and $\left.\Delta C_{\text {deoxy }(\lambda 1, \lambda 2)}^{\prime}\right)$ are expressed as changes in the concentration $\left(C_{\text {oxy }}\right.$ and $\left.C_{\text {deoxy }}\right)$ multiplied by the indefinite optical path length $(L)$ in the activation region, where $\Delta A, \varepsilon_{\text {oxy }}$, and $\varepsilon_{\text {deoxy }}$ indicate the change in optical density of the detected light, the extinction coefficient of oxy- $\mathrm{Hb}$, and that of deoxy- $\mathrm{Hb}$, respectively, for two wavelengths $(\lambda 1$ and $\lambda 2)$. We assume that $L$ is equal for every wavelength and constant. ${ }^{23}$

We defined a period from $5 \mathrm{~s}$ prior to each task condition period to the end of the following control condition period as the analysis block. Each session consisted of five analysis blocks. Then, we applied linear regression by the least squares method to the data in each analysis block during the first 5-s 


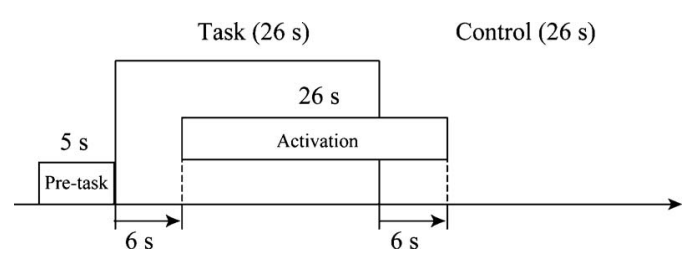

Fig. 4 Pretask and activation periods in the analysis block. Assuming that the hemodynamic changes induced by the task were delayed $6 \mathrm{~s}$ from the task onset, the shifted period of the task condition period was defined as the activation period. A 5-s period prior to each task condition period was defined as the pretask period. Mean values during the pretask period and the activation period were used for statistical analysis.

period and the last 1 -s period to determine the linear trend of the baseline unrelated to the behavioral task. After correcting the baseline by removing the trend, we averaged the baselinecorrected data in all the analysis blocks.

$\mathrm{The} \mathrm{Hb}$ signals were statistically assessed. Assuming that the hemodynamic changes induced by the AD task were delayed $6 \mathrm{~s}$ from the task onset, the shifted period of the task condition period was defined as the activation period, ${ }^{23}$ and a 5 -s period prior to each task condition period was defined as the pretask period (Fig. 4). Using the averaged $\mathrm{Hb}$ signals $\left(\Delta C_{\text {oxy }}^{\prime}, \Delta C_{\text {deoxy }}^{\prime}\right)$ during the pretask period and those during the activation period for each analysis block, we calculated the $t$ value (paired $t$ test) between the averaged $\mathrm{Hb}$ signals in the pretask periods of five analysis blocks, and the $\mathrm{Hb}$ signals in the activation periods of the same five blocks.

We used the probabilistic estimation method ${ }^{24}$ to register the OT measurement data of the Montreal Neurological Institute (MNI) standard brain space. Irradiation and detection positions, together with several scalp landmarks, were digitized using a 3-D magnetic space digitizer (Isotrak II Polhemus, Colchester, Vermont). We obtained the mean estimate for the location of measurement points for ten volunteers ( 5 males and 5 females, including 6 subjects for OT measurement).

\section{Results}

\subsection{Time Course of Oxy- and Deoxyhemoglobin Concentrations}

The time course of oxy- and deoxy-Hb signals $\left(\Delta C_{\text {oxy }}^{\prime}\right.$ and $\Delta C_{\text {deoxy }}^{\prime}$, respectively) obtained from channel 8 (Fig. 1) for a representative subject are shown in Fig. 5(a). During the task condition periods, $\Delta C_{\text {oxy }}^{\prime}$ increased and $\Delta C_{\text {deoxy }}^{\prime}$ decreased a little, which shows task-related and reproducible changes of $\Delta C_{\text {oxy }}^{\prime}$. To assess the task-related and step-related changes, power spectra of the data were calculated [Fig. 5(b)]. In the $\Delta C_{\text {oxy }}^{\prime}$ spectrum, the task-related frequency is $1.92 \mathrm{E}-2$ ( $=1 / 52) \mathrm{Hz}$, because one cycle of a task condition and a control condition is $52 \mathrm{~s}$, and the step-related frequency is $2 \mathrm{~Hz}$. The power at the former frequency is $26 \mathrm{~dB}$ larger than that at the latter, which is low enough to obtain the task-related changes. By use of the moving-averaged data, the power at the step-related frequency becomes $61 \mathrm{~dB}$ smaller than that at the data before the moving average, so the step-related changes are small enough to be negligible. The same can be said for $\Delta C_{\text {deoxy }}^{\prime}$.

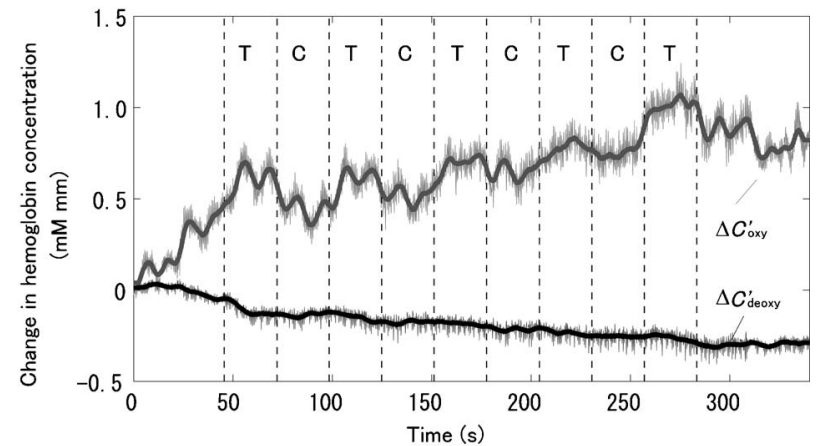

(a)

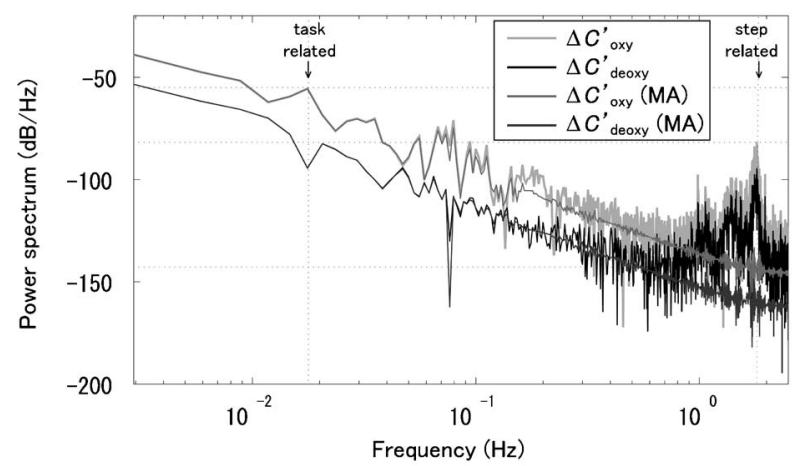

(b)

Fig. 5 (a) Time-series data of oxy- and deoxy- $\mathrm{Hb}$ signals obtained from channel 8 [Fig. 1(a)] for a representative subject. Light and dark gray lines represent the oxy- and deoxy-Hb signals $\left(\Delta C_{\text {oxy }}^{\prime}\right.$ and $\left.\Delta C_{\text {deoxy }}^{\prime}\right)$, respectively, and thick lines represent moving-averaged data for $5 \mathrm{~s}$. Vertical dashed lines show the onset of a condition change. T: task condition and C: control condition. (b) Power spectra of the oxyand deoxy $\mathrm{Hb}$ signals shown in (a). Arrows show the task-related and step-related frequencies. MA is the moving-averaged data.

To examine the task-related changes in $\Delta C_{\text {oxy }}^{\prime}$ and $\Delta C_{\text {deoxy }}^{\prime}$, we corrected the baseline to remove low-frequency fluctuations caused by laser drifts or biological metabolism for each analysis block, then we averaged the baseline-corrected data of all the blocks without low signal-to-noise ratio blocks. The mean time courses of block-averaged $\Delta C_{\text {oxy }}^{\prime}$ and $\Delta C_{\text {deoxy }}^{\prime}$ for each channel obtained from all the subjects are shown in Fig. 6. Large changes in $\Delta C_{\mathrm{oxy}}^{\prime}$ during the task condition period were observed in channels 3 through 16, which were positioned from the center to the right part of the prefrontal area [Fig. 1(a)], and small changes in $\Delta C_{\text {deoxy }}^{\prime}$ were observed as reported in the previous studies. ${ }^{15-17}$ To assess the task-related changes in $\Delta C_{\mathrm{oxy}}^{\prime}$ and $\Delta C_{\text {deoxy }}^{\prime}$ statistically, we calculated the $t$ value (paired $t$ test) between the mean changes in those $\mathrm{Hb}$ signals in the pretask periods and those in the activation periods (Fig. 4). Significant $t$ values (two-tailed $t$ test, $p$ $<0.001)$ were obtained in $\Delta C_{\text {oxy }}^{\prime}$ in channels 5 through 10 , 13, and 15 (Fig. 7), but no significant $t$ values were obtained in $\Delta C_{\text {deoxy }}^{\prime}$. We identified the measurement points with significant $t$ values in $\Delta C_{\text {oxy }}^{\prime}$ as activation channels.

\section{Discussion}

In the time course of oxy- and deoxy-Hb signals [Fig. 5(a)], the task-related changes in the oxy-Hb signal were observed 


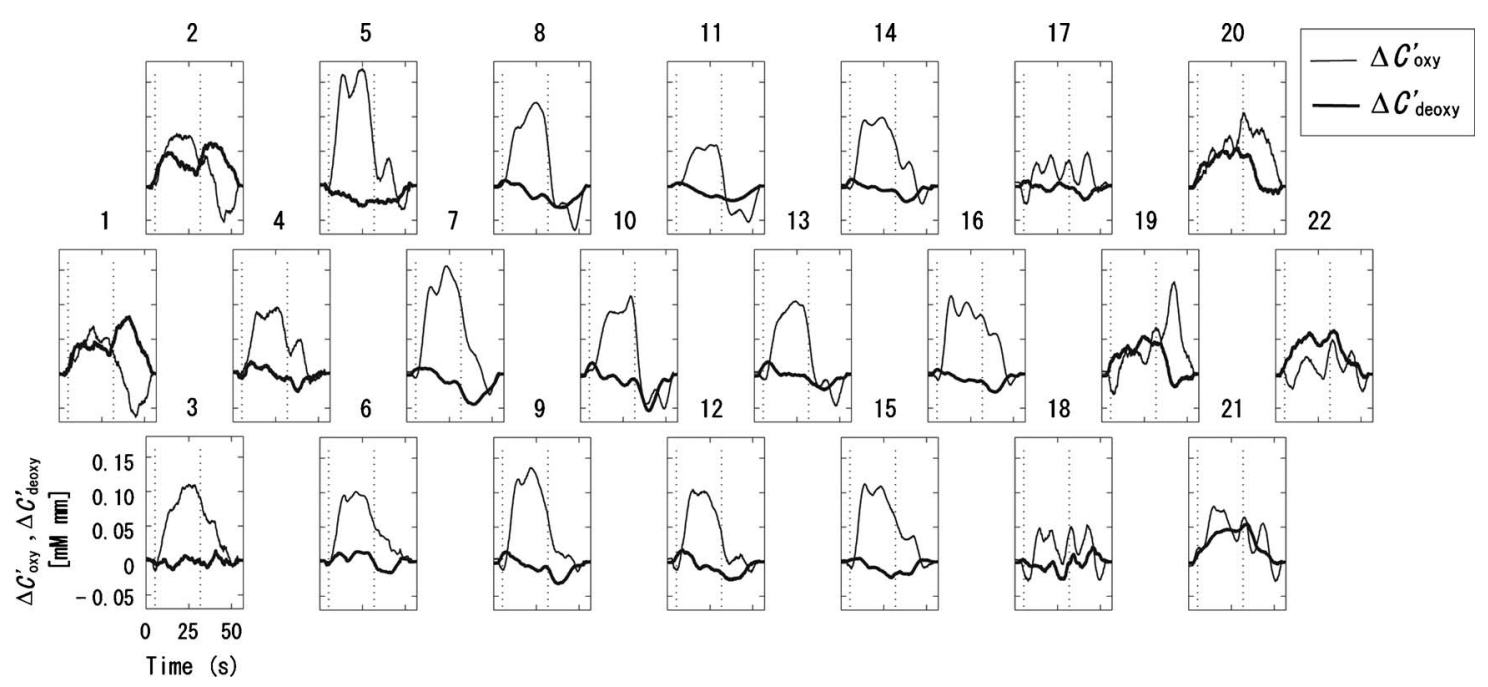

Fig. 6 Mean time-series data of all subjects for block-averaged oxy- and deoxy-Hb signals for each measurement channel. Thin and thick lines represent oxy- and deoxy-Hb signals, respectively. Vertical dashed lines represent task condition periods.

with high-frequency changes. As shown by the power spectrum in Fig. 5(b), the step-related changes were small enough to be negligible, especially in the moving-averaged data. These results suggest that the probe unit was well attached to the subject's head by its flexible design [see Fig. 1(b)], so it could produce an effect of reducing movement artifacts. Thus, the WOT system is effective for OT measurement of the prefrontal sites during natural walking.

The block-averaged data shown in Fig. 6 and statistical analysis shown in Fig. 7 indicate that the center and the right area of the prefrontal cortex were activated during the AD task used in the current study. To confirm where the statistically significant activation areas shown in Fig. 7 are positioned in MNI standard brain space, we used the probabilistic estimation method ${ }^{24}$ (see Sec. 2) and obtained the mean estimate for the location of measurement points for ten volunteers (Fig. 8 and Table 1). Figure 8 and Table 1 indicate that channels 5 through 8 cover an area around the dorsolateral prefrontal cortex (DLPFC) in the right hemisphere, and that channels 9, 10, 13, and 15 cover the rostral prefrontal area, including the Brodmann area 10 (BA 10). The DLPFC plays important roles in working memory ${ }^{25,26}$ and in attention allocation in conflict processing. ${ }^{27,28}$ The AD task in the current study required constant allocation of attention to a ball and motor control to balance the ball on a card, as shown by the visual and motor feedback in the task condition, which must have activated DLPFC. The role of BA 10 in the rostral prefrontal area in performing a cognitive branching task that requires the maintenance of a primary task while simultaneously allocating attention to another task has recently been

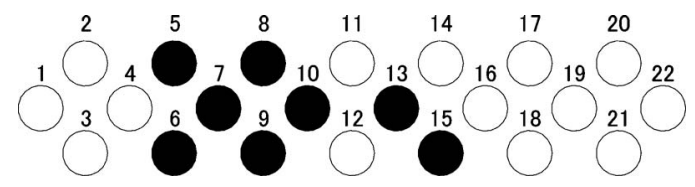

Fig. 7 Measurement channels showing significant $\Delta C_{\text {oxy }}^{\prime}$ (two-tailed $t$ test, $p<0.001)$. Black circles show significant channels. discussed. $^{29,30}$ Our behavioral task, where stepping the right foot in time to a sound while walking was always required in a session and simultaneously balancing a ball on a card was also required in the task condition, activated the rostral prefrontal area as a cognitive branching task.

This study showed that the WOT system was applicable to assessment of large prefrontal areas, including the right and left DLPFC and BA 10, by simultaneously measuring the $\mathrm{Hb}$ signals of these areas. Recent studies have reported wearable NIRS or OT systems and were successful in freeing up the subject's posture, ${ }^{19-21}$ so these systems could be available in similar situations as used in this study. However, they had two to eight measurement points covering a region that was much less than or comparable to half of the forehead. The WOT system in this study has an advantage of studying various prefrontal functions over the other systems.

We demonstrated in the present study that the cognitive functions of subjects who performed $\mathrm{AD}$ tasks while walking

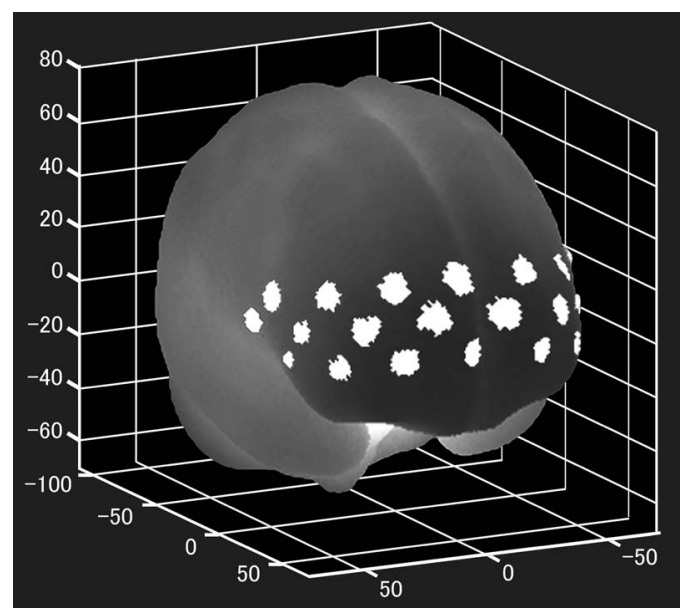

Fig. 8 Mean estimate for location of measurement points in MNI standard brain space for ten volunteers. Measurement points cover area around the dorsolateral prefrontal cortex and rostral prefrontal area. 
Table 1 MNI coordinates of estimated cortical projection points for all measurement channels.

\begin{tabular}{|c|c|c|c|}
\hline & \multicolumn{3}{|c|}{ MNI coordinates } \\
\hline & $x$ & $y$ & $z$ \\
\hline Chl & 63.6 & 13.4 & 11.3 \\
\hline Ch2 & 60.7 & 22.7 & 21.4 \\
\hline Ch3 & 58.1 & 31.9 & 0.2 \\
\hline Ch4 & 56.4 & 37.9 & 11.1 \\
\hline Ch5 & 49.0 & 44.2 & 24.6 \\
\hline Ch6 & 48.8 & 53.1 & 0.6 \\
\hline Ch7 & 41.1 & 58.7 & 14.6 \\
\hline Ch8 & 30.2 & 59.2 & 28.1 \\
\hline Ch9 & 30.8 & 67.9 & 4.4 \\
\hline Ch10 & 19.8 & 69.5 & 18.7 \\
\hline Ch11 & 7.2 & 64.5 & 30.7 \\
\hline Ch12 & 5.7 & 72.3 & 5.3 \\
\hline $\mathrm{Ch} 13$ & -9.0 & 70.0 & 17.9 \\
\hline $\mathrm{Ch} 14$ & -20.8 & 61.4 & 29.6 \\
\hline Ch 15 & -22.9 & 69.3 & 2.9 \\
\hline $\mathrm{Ch} 16$ & -35.4 & 61.0 & 14.1 \\
\hline Ch17 & -43.5 & 46.7 & 25.9 \\
\hline Ch18 & -44.7 & 54.6 & -0.8 \\
\hline Ch19 & -52.3 & 40.2 & 10.7 \\
\hline Ch2O & -57.4 & 23.1 & 22.3 \\
\hline Ch21 & -55.1 & 34.2 & -1.7 \\
\hline Ch22 & -60.8 & 14.3 & 9.6 \\
\hline
\end{tabular}

could be evaluated. Studies on the human ability to walk while performing AD tasks by using behavioral methods ${ }^{31-33}$ have analyzed walking parameters. These studies could be highly important for assessing and preventing mobility loss in older adults, and our functional imaging technique with WOT will be a powerful tool for studies in these areas.

To generalize the present study, we need to design more precise paradigms and to investigate more subjects for discussing details of cognitive functions, especially those related to a subject's physical ability, age, or gender. However, we believe that our experiment has shown that the WOT system is useful for studying cognitive functions of moving subjects.

\section{Conclusion}

We demonstrate that the wearable optical topography (WOT) system is capable of measuring oxy- and deoxy-Hb signals in the human cerebral cortex during an AD task performed while walking. In the time course of the oxy- and deoxy-Hb signals, we observe both task- and step-related changes, but the latter changes are negligible compared to the former. Thus, the result shows that the WOT system is robust enough during walking to obtain task-related changes. Statistical assessment of the task-related changes in $\Delta C_{\text {oxy }}^{\prime}$ using a two-tailed $t$ test $(p<0.001)$ shows that DLPFC and the rostral prefrontal area are significantly activated during performance of the AD task while walking, which suggests that the task is a cognitive branching task. These results show that our functional imaging technique with the WOT system will be a useful tool for evaluating brain activation related to cognitive tasks performed while walking.

\section{References}

1. A. Villringer, J. Planck, C. Hock, L. Schleinkofer, and U. Dirnagl, "Near infrared spectroscopy (NIRS): a new tool to study hemodynamic changes during activation of brain function in human adults," Neurosci. Lett. 154(1-2), 101-104 (1993).

2. T. Kato, A. Kamei, S. Takashima, and T. Ozaki, "Human visual cortical function during photic stimulation monitoring by means of nearinfrared spectroscopy," J. Cereb. Blood Flow Metab. 13(3), 516-520 (1993).

3. Y. Hoshi and M. Tamura, "Detection of dynamic changes in cerebral oxygenation coupled to neuronal function during mental work in man," Neurosci. Lett. 150(1), 5-8 (1993).

4. B. Chance, Z. Zhuang, C. UnAh, C. Alter, and L. Lipton, "Cognitionactivated low-frequency modulation of light absorption in human brain," Proc. Natl. Acad. Sci. U.S.A. 90(8), 3770-3774 (1993).

5. A. Maki, Y. Yamashita, Y. Ito, E. Watanabe, Y. Mayanagi, and H. Koizumi, "Spatial and temporal analysis of human motor activity using noninvasive NIR topography," Med. Phys. 22(12), 1997-2005 (1995).

6. Y. Yamashita, A. Maki, Y. Ito, E. Watanabe, H. Mayanagi, and H. Koizumi, "Noninvasive near-infrared topography of human brain activity using intensity modulation spectroscopy," Opt. Eng. 35(4), 1046-1099 (1996).

7. A. Maki, Y. Yamashita, E. Watanabe, and H. Koizumi, "Visualizing human motor activity by using non-invasive optical topography," Front Med. Biol. Eng. 7(4), 285-297 (1996).

8. H. Koizumi, Y. Yamashita, A. Maki, T. Yamamoto, Y. Ito, H. Itagaki, and R. P. Kennan, "Higher-order brain function analysis by transcranial dynamic near-infrared spectroscopy imaging," J. Biomed. Opt. 4(4), 403-413 (1999).

9. G. Taga, K. Asakawa, A. Maki, Y. Konishi, and H. Koizumi, "Brain imaging in awake infants by near-infrared optical topography," Proc. Natl. Acad. Sci. U.S.A. 100(19), 10722-10727 (2003).

10. M. Pena, A. Maki, D. Kovacic, G. Dehaene-Lambertz, H. Koizumi, F. Bouquet, and J. Mehler, "Sounds and silence: an optical topography study of language recognition at birth," Proc. Natl. Acad. Sci. U.S.A. 100(20), 11702-11705 (2003).

11. T. Kusaka, K. Kawada, K. Okubo, K. Nagano, M. Namba, H. Okada, T. Imai, K. Isobe, and S. Itoh, "Noninvasive optical imaging in the visual cortex in young infants," Hum. Brain Mapp 22, 122-132 (2004).

12. H. Bortfeld, E. Wruck, and D. A. Boas, "Assessing infants' cortical response to speech using near-infrared spectroscopy," Neuroimage 34, 407-415 (2007).

13. E. Watanabe, A. Maki, F. Kawaguchi, K. Takashiro, Y. Yamashita, H. Koizumi, and Y. Mayanagi, "Non-invasive assessment of language dominance with near-infrared spectroscopic mapping," Neurosci. Lett. 256(1), 49-52 (1998).

14. M. Kameyama, M. Fukuda, Y. Yamagishi, T. Sato, T. Uehara, M. Ito, T. Suto, and M. Mikuni, "Frontal lobe function in bipolar disorder: a multichannel near-infrared spectroscopy study," Neuroimage 29, 172-184 (2006).

15. I. Miyai, H. C. Tanabe, I. Sase, H. Eda, I. Oda, I. Konishi, Y. Tsunazawa, T. Suzuki, T. Yanagida, and K. Kubota, "Cortical mapping of gait in humans: a near-infrared spectroscopic topography study," Neuroimage 14, 1186-1192 (2001). 
16. M. Suzuki, I. Miyai, T. Ono, I. Oda, I. Konishi, T. Kochiyama, and K. Kubota, "Prefrontal and premotor cortices are involved in adapting walking and running speed on the treadmill: an optical imaging study," Neuroimage 23, 1020-1026 (2004).

17. M. Suzuki, I. Miyai, T. Ono, and K. Kubota, "Activities in the frontal cortex and gait performance are modulated by preparation. An fNIRS study," Neuroimage 39, 600-607 (2008).

18. M. Mihara, I. Miyai, M. Hatakenaka, K. Kubota, and S. Sakoda, "Role of the prefrontal cortex in human balance control," Neuroimage 43, 329-336 (2008).

19. Y. Hoshi, S. J. Chen, and M. Tamura, "Spatiotemporal imaging of human brain activity by functional near-infrared spectroscopy," Am. Lab. (Boston) 33, 35-39 (2001).

20. G. Yurtsever, A. Bozkurt, F. Kepics, K. Pourrezaei, and A. Devaraj, "Pocket PC based wireless continuous wave near infrared spectroscopy system for functional imaging of human brain," Proc. 25th Ann. Intl. Conf. IEEE Eng. Med. Biol. Soc., pp. 3435-3437 (2003).

21. T. Muehlemann, D. Haensse, and M. Wolf, "Wireless miniaturized in-vivo near infrared imaging," Opt. Express 16(14), 10323 (2008).

22. H. Atsumori, M. Kiguchi, A. Obata, H. Sato, T. Katura, T. Funane, and A. Maki, "Development of wearable optical topography system for mapping the prefrontal cortex activation," Rev. Sci. Instrum. 80, 043704 (2009).

23. H. Sato, M. Kiguchi, F. Kawaguchi, and A. Maki, "Practicality of wavelength selection to improve signal-to-noise ratio in near-infrared spectroscopy," Neuroimage 21, 1554-1562 (2004).

24. A. K. Singh, M. Okamoto, H. Dan, V. Jurcak, and I. Dan, "Spatial registration of multichannel multi-subject fNIRS data to MNI space without MRI," Neuroimage 27, 842-851 (2005).

25. B. R. Postle, C. E. Stern, B. R. Rosen, and S. Corkin, "An fMRI investigation of cortical contributions to spatial and nonspatial visual working memory," Neuroimage 11, 409-423 (2000).

26. S. Tsujimoto, T. Yamamoto, H. Kawaguchi, H. Koizumi, and T. Sawaguchi, "Prefrontal cortical activation associated with working memory in adults and preschool children: an event-related optical topography study," Cereb. Cortex 14(7), 703-712 (2004).

27. M. P. Milham, M. T. Banich, E. D. Claus, and N. J. Cohen, "Practicerelated effects demonstrate complementary roles of anterior cingulated and prefrontal cortices in attentional control," Neuroimage $\mathbf{1 8}$ 483-493 (2003).

28. T. L. Luks, G. V. Simpson, C. L. Dale, and M. G. Hough, "Preparatory allocation of attention and adjustments in conflict processing," Neuroimage 35, 949-958 (2007).

29. E. Koechlin, G. Basso, P. Pietrini, S. Panzer, and J. Grafman, "The role of the anterior prefrontal cortex in human cognition," Nature (London) 399, 148-151 (1999).

30. T. S. Braver and S. R. Bongiolatti, "The role of frontopolar cortex in subgoal processing during working memory," Neuroimage 15, 523536 (2002).

31. P. C. Grabiner, S. T. Biswas, and M. D. Grabiner, "Age-related changes in spatial and temporal gait variables," Arch. Phys. Med. Rehabil. 82, 31-35 (2001).

32. A. K. Coppin, A. Shumway-Cook, J. S. Saczynski, K. V. Patel, A. Ble, L. Ferrucci, and J. M. Guralnik, "Association of executive function and performance of dual-task physical tests among older adults: analyses from the InChianti study," Ageing 35, 619-624 (2006).

33. O. Beauchet, V. Dubost, F. R. Herrmann, and R. W. Kressig, "Strideto-stride variability while backward counting among healthy young adults," J. Neuroeng. Rehabil. 2, 26 (2005). 